\title{
DING PROJECTIVE AND DING INJECTIVE DIMENSIONS
}

\author{
Chaoling Huang and Tongsuo Wu \\ Received: 5 September 2013; Revised: 26 January 2015 \\ Communicated by Sait Halıcıŏ̆lu
}

\begin{abstract}
The concepts of Ding projective, injective $R$-modules were first introduced by Ding and his partners in [J. Aust. Math. Soc., 86 (2009), 323338] and [J. Algebra Appl., 7(4) (2008), 491-506], respectively. In this paper, we investigate Ding projective, Ding injective dimensions of modules and rings.

Mathematics Subject Classification (2010): 16D40, 16E05, 18G20

Keywords: Gorenstein projective module, Gorenstein injective module, Ding projective module, Ding injective module, change of rings
\end{abstract}

\section{Introduction}

Recall that an $R$-module $M$ is Gorenstein projective (for short $G$-projective; see [6]) if there is an exact sequence

$$
\mathbf{P}=\cdots \rightarrow P_{1} \rightarrow P_{0} \rightarrow P^{0} \rightarrow P^{1} \rightarrow \cdots
$$

of projective modules with $M=\operatorname{Ker}\left(P^{0} \rightarrow P^{1}\right)$ such that $\operatorname{Hom}(\mathbf{P}, Q)$ is exact for each projective $R$-module $Q$. Such exact sequence is called a complete projective resolution. The class of all Gorenstein projective $R$-modules is denoted by $\mathcal{G P}(R)$. Enochs and Jenda in [6] defined a homological dimension, namely the Gorenstein projective dimension, $G p d_{R}(-)$, for any $R$-module. We say that $M$ has Gorenstein projective dimension at most $n$, denoted $\operatorname{Gpd}_{R}(M) \leq n$, if there is a Gorenstein projective resolution, i.e., there is an exact sequence $0 \rightarrow G_{n} \rightarrow \cdots \rightarrow G_{0} \rightarrow M \rightarrow$ 0 , where all $G_{i}$ are $G$-projective $R$-modules, and say $G_{p d}(M)=n$ if there is not a shorter Gorenstein projective resolution. Dually, one can consider $G$-injectives; see [6]. Analogously, one can consider $G$-flats; see [8]. The class of all Gorenstein injective (flat) $R$-modules is denoted by $\mathcal{G I}(R)(\mathcal{G F}(R))$; for any $R$-module $M$, the Gorenstein injective (flat) dimension is denoted by $\operatorname{Gid}_{R}(M)\left(G f d_{R}(M)\right)$. The reader can refer to $[4,7,12,11]$ for more details about Gorenstein projective, injective and flat modules.

Gillespie in [10] defined three classes of $R$-modules which are called Ding injective, Ding projective and Ding flat, respectively and developed their standard model

The second author is partially supported by the NSFC (No. 11271250). 
structures on ${ }_{R} \mathcal{M}$. Yang and his coauthors in $[24,22]$ researched the homological properties of Ding injective, Ding projective $R$-modules. Ding projective modules were first introduced by Ding, Li and Mao in [5] where they were called strongly Gorenstein flat modules. Ding injective modules were first introduced by Ding and Mao in [16] as Gorenstein FP-injective modules.

In this paper, we consider Ding projective and Ding injective modules and dimensions. It is organized as follows: In Section 2 we collect some fundamental definitions and facts for using later. Section 3 is first devoted to the study of the Ding projective and Ding injective modules and dimensions under change of rings. Recall that if $\mathcal{C}$ and $\mathcal{G}$ are classes of objects of an abelian category $\mathcal{A}$, then we say $\operatorname{Hom}(-,-)$ is right $\mathcal{C} \times \mathcal{G}$ balanced if for objects $X$ and $Y$ of $\mathcal{A}$ there exist complexes

$$
\cdots \rightarrow C_{1} \rightarrow C_{0} \rightarrow X \rightarrow 0
$$

and

$$
0 \rightarrow Y \rightarrow G^{0} \rightarrow G^{1} \rightarrow \cdots
$$

such that $C_{i} \in \mathcal{C}$ and $G^{i} \in \mathcal{G}$ for all $i \geq 0$ and such that $\operatorname{Hom}(-, \mathcal{G})$ makes the first complex exact and $\operatorname{Hom}(\mathcal{C},-)$ makes the second complex exact. The author in $[22$, Theorem 3.6] proved that $\operatorname{Hom}(-,-)$ is right balance by $\mathcal{D P}(R) \times \mathcal{D} \mathcal{I}(R)$ for any Ding-Chen ring $R$. Then we give the result a new proof. At last in Section 3 , we give the new characterizations to finite $D$-projective dimension of $R$-modules as follows.

Theorem. (Theorem 3.13 and 3.15) Let $M$ be an R-module and $n$ be a nonnegative integer. Then the following are equivalent.

(1) $\operatorname{Dpd}_{R}(M) \leq n$.

(2) For some integer $k$ with $1 \leq k \leq n$, there is an exact sequence $0 \rightarrow P_{n} \rightarrow$ $\cdots \rightarrow P_{1} \rightarrow P_{0} \rightarrow M \rightarrow 0$ such that $P_{i}$ is D-projective if $0 \leq i<k$ and $P_{j}$ is projective if $j \geq k$.

(3) For any integer $k$ with $1 \leq k \leq n$, there is an exact sequence $0 \rightarrow P_{n} \rightarrow$ $\cdots \rightarrow P_{1} \rightarrow P_{0} \rightarrow M \rightarrow 0$ such that $P_{i}$ is $D$-projective if $0 \leq i<k$ and $P_{j}$ is projective if $j \geq k$.

$\left(2^{\prime}\right)$ For some integer $k$ with $0 \leq k \leq n$, there is an exact sequence $0 \rightarrow A_{n} \rightarrow$ $\cdots \rightarrow A_{1} \rightarrow A_{0} \rightarrow M \rightarrow 0$ such that $A_{k}$ is D-projective and other $A_{i}$ projective.

$\left(3^{\prime}\right)$ For any integer $k$ with $0 \leq k \leq n$, there is an exact sequence $0 \rightarrow A_{n} \rightarrow$ $\cdots \rightarrow A_{1} \rightarrow A_{0} \rightarrow M \rightarrow 0$ such that $A_{k}$ is D-projective and other $A_{i}$ projective.

Recall that the second change of rings theorem for the projective and injective dimensions as follows (see, [19, Theorem 4.3.5 and Exercise 4.3.3]): Let $x$ be a 
central nonzerodivisor in a ring $R$. If $A$ is an $R$-module and $x$ is a nonzerodivisor on $A$, then $p d_{R}(A) \geq p d_{R / x}(A / x A)$ and $i d_{R}(A) \geq 1+i d_{R / x}(A / x A)$ if $A$ is not injective. Bennis and Mahdou in [2] proved the second change of rings theorem for the Gorenstein projective and injective dimensions. In Section 4 we investigate the strongly $D$-projective and $D$-injective modules and the second change of rings theorem for $D$-projective and $D$-injective dimensions.

Setup and notation. Throughout this paper, $R$ and $S$ are associative rings with identity and all modules are unitary. ${ }_{R} \mathcal{M}$ denotes the category of left $R$ modules, and $\mathcal{P}(R), \mathcal{I}(R)$ and $\mathcal{F}(R)$ denotes the class of projective, injective and flat modules, respectively. We denote by ${ }_{R} M$ a left $R$-module, $p d(M), i d(M)$ and $f d(M)$ stand for the projective, injective and flat dimensions of $M$, respectively. We write $l D(R)(w D(R))$ for the left (weak) global dimension of $R$.

\section{Preliminaries}

In this section, we recall some definitions and collect some fundamental results.

Definition 2.1. Let $S$ be a ring and let R be a subring of $S$ (with the same 1). $S$ is called a finite normalizing extension of $R$ if there exist elements $a_{1}, \ldots, a_{n} \in S$ such that $a_{1}=1, S=R a_{1}+\cdots+R a_{n}$ and $R a_{i}=a_{i} R$ for all $i=1, \cdots, n . S$ is called a free normalizing extension of $\mathrm{R}$ if $a_{1}=1, S=R a_{1}+\cdots+R a_{n}$ is a finite normalizing extension and $S$ is free with basis $\left\{a_{1}, \ldots, a_{n}\right\}$ as an $R$-module. $S$ is said to be an excellent extension of $R$ in case $S$ is a free normalizing extension of $R$ and $S$ has $R$-projectivity (that is, if ${ }_{S} M$ is an $S$-module and ${ }_{S} N$ is a submodule of ${ }_{S} M$, then ${ }_{R} N \mid{ }_{R} M$ implies ${ }_{S} N \mid{ }_{S} M$, where $N \mid M$ means $N$ is a direct summand of $M)$.

Lemma 2.2. [21, Lemma 1.1] Let $S \geq R$ be a ring extension such that $S$ has $R$ projectivity. If $M$ is a left $S$-module, then ${ }_{S} M$ is isomorphic to a direct summand of ${ }_{S}\left(S \otimes_{R} M\right)$ and ${ }_{S} \operatorname{Hom}_{R}(S, M)$.

Recall that a left $R$-module $M$ is called $F P$-injective if $\operatorname{Ext}_{R}^{1}(N, M)=0$ for all finitely presented left $R$-module $N$.

Definition 2.3. (1) ([10, Definition 3.6]) We call a left $R$-module $M$ Ding projective (for short, $D$-projective) if there is an exact sequence

$$
\mathbf{P}=\cdots \rightarrow P_{1} \rightarrow P_{0} \rightarrow P^{0} \rightarrow P^{1} \rightarrow \cdots
$$

of projective modules with $M=\operatorname{Ker}\left(P^{0} \rightarrow P^{1}\right)$ such that $\operatorname{Hom}(\mathbf{P}, Q)$ is exact for each flat $R$-module $Q$. The class of all $D$-projective modules is denoted by $\mathcal{D P}(R)$. 
(2) ([10, Definition 3.2]) We call a left $R$-module $M$ Ding injective (for short, $D$-injective) if there is an exact sequence

$$
\mathbf{I}=\cdots \rightarrow I_{1} \rightarrow I_{0} \rightarrow I^{0} \rightarrow I^{1} \rightarrow \cdots
$$

of injective modules with $M=\operatorname{Ker}\left(I^{0} \rightarrow I^{1}\right)$ such that $\operatorname{Hom}(E, \mathbf{I})$ is exact for each $F P$-injective $R$-module $E$. The class of all $D$-injective modules is denoted by $\mathcal{D I}(R)$.

From Definition 2.3 one can obtain the following characterization of $D$-projective and injective $R$-modules.

Proposition 2.4. (1) $M$ is D-projective if and only if $\operatorname{Ext}_{R}^{n \geq 1}(M, Q)=0$ and there exists an exact sequence of the form:

$$
X=0 \rightarrow M \rightarrow P^{0} \rightarrow P^{1} \rightarrow \cdots
$$

such that $\operatorname{Hom}_{R}(X, Q)$ is exact for any flat $R$-module $Q$.

(2) $M$ is D-injective if and only if $\operatorname{Ext}_{R}^{n \geq 1}(J, M)=0$ and there exists an exact sequence of the form:

$$
Y=\cdots \rightarrow I_{1} \rightarrow I_{0} \rightarrow M \rightarrow 0
$$

such that $\operatorname{Hom}_{R}(J, Y)$ is exact for any $F P$-injective $R$-module $J$.

Definition 2.5. For any $R$-module $M$, the $D$-projective dimension of $M$, denoted by $\operatorname{Dpd}_{R}(M)$ is defined as the infimum of the set of $n$ such that there exists an exact sequence $0 \rightarrow G_{n} \rightarrow \cdots \rightarrow G_{0} \rightarrow M \rightarrow 0$ of left $R$-modules, where all $G_{i}$ are $D$-projective $R$-modules. If no such $n$ exists, set $D p d_{R}(M)=\infty$.

Similarly, we can define the $D$-injective dimension of $R$-module $M$, denoted by $\operatorname{Did}_{R}(M)$.

Recall that in [11] a class $\mathcal{X}$ of modules is projectively resolving if $\mathcal{P}(R) \subseteq \mathcal{X}$, and for every short exact sequence $0 \rightarrow X^{\prime} \rightarrow X \rightarrow X^{\prime \prime} \rightarrow 0$ with $X^{\prime \prime} \in \mathcal{X}$ the conditions $X^{\prime} \in \mathcal{X}$ and $X \in \mathcal{X}$ are equivalent; $\mathcal{X}$ is injectively resolving if $\mathcal{I}(R) \subseteq \mathcal{X}$, and for every short exact sequence $0 \rightarrow X^{\prime} \rightarrow X \rightarrow X^{\prime \prime} \rightarrow 0$ with $X^{\prime} \in \mathcal{X}$ the conditions $X \in \mathcal{X}$ and $X^{\prime \prime} \in \mathcal{X}$ are equivalent.

Proposition 2.6. (1) [24, Theorem 2.6 and Corollary 2.7] The class of D-projective $R$-modules is projectively resolving and closed under direct summands.

(2) [24, Theorem 2.8 and Corollary 2.9] The class of D-injective R-modules is injectively resolving and closed under direct summands. 


\section{3. $D$-projective modules and $D$-injective modules}

In this section, we first develop properties of $D$-projective ( $D$-injective) modules and dimensions under change of rings.

Proposition 3.1. Let $\theta: R \rightarrow S$ be a ring homomorphism with ${ }_{R} S$ flat. If $M$ is a $D$-projective $R$-module, then $S \otimes_{R} M$ is a D-projective $S$-module.

Proof. Since $M$ is a $D$-projective $R$-module, there is an exact sequence of projective $R$-modules:

$$
P=\cdots \rightarrow P_{1} \rightarrow P_{0} \rightarrow P^{0} \rightarrow P^{1} \rightarrow \cdots
$$

Since $S$ is flat,

$$
S \otimes_{R} P=\cdots \rightarrow S \otimes_{R} P_{1} \rightarrow S \otimes_{R} P_{0} \rightarrow S \otimes_{R} P^{0} \rightarrow S \otimes_{R} P^{1} \rightarrow \cdots
$$

is exact and $S \otimes_{R} M \cong \operatorname{Ker}\left(S \otimes_{R} P_{1} \rightarrow S \otimes_{R} P_{0}\right)$. On the other hand, for any flat $S$-module $Q$, it is also a flat $R$-module.

$$
\begin{aligned}
\operatorname{Hom}_{S}\left(S \otimes_{R} P, Q\right) & \cong \operatorname{Hom}_{R}\left(P, \operatorname{Hom}_{S}(S, Q)\right) \\
& \cong \operatorname{Hom}_{R}(P, Q) .
\end{aligned}
$$

Thus $\operatorname{Hom}_{S}\left(S \otimes_{R} P, Q\right)$ is exact, therefore, $S \otimes_{R} M$ is a $D$-projective $S$-module.

Corollary 3.2. Let $\theta: R \rightarrow S$ be a ring homomorphism with ${ }_{R} S$ flat and let $M$ be an $R$-module. Then $\operatorname{Dpd}_{S}\left(S \otimes_{R} M\right) \leq \operatorname{Dpd}_{R}(M)$.

Corollary 3.3. Let $R[x]$ be a polynomial ring in one variable over $R$. For any $R$-module $M$, denote $R[x]$-module $R[x] \otimes_{R} M$ by $M[x]$. Then $\operatorname{Dpd}_{R[x]}(M[x]) \leq$ $\operatorname{Dpd}_{R}(M)$.

Proof. Since $R[x]$ is a flat $R$-module, it is true by Corollary 3.2 .

Theorem 3.4. Let $S \geq R$ be an excellent extension of $R$ and let $M$ be an $R$ module. $M$ is a D-projective $R$-module if and only if $S \otimes_{R} M$ is a D-projective $S$-module.

Proof. $\Rightarrow$ By Proposition 3.1.

$\Leftarrow$ For any flat $R$-module $Q, S \otimes_{R} Q$ is a flat $S$-module. Since $S$ is an excellent extension of $R, S \cong R^{n}$ as $R$-modules for some integer $n$.

$$
\begin{aligned}
\operatorname{Ext}_{R}^{j \geq 1}\left(M^{n}, Q\right) & \cong \operatorname{Ext}_{R}^{j \geq 1}\left(M, S \otimes_{R} Q\right) \\
& \cong \operatorname{Ext}_{R}^{j \geq 1}\left(M, \operatorname{Hom}_{S}\left(S, S \otimes_{R} Q\right)\right. \\
& \cong \operatorname{Ext}_{S}^{j \geq 1}\left(S \otimes_{R} M, S \otimes_{R} Q\right)=0,
\end{aligned}
$$

since $S \otimes_{R} M$ is a $D$-projective $S$-module. On the other hand, there exists an exact sequence of the form:

$$
{ }_{S} X=0 \rightarrow S \otimes_{R} M \rightarrow P^{0} \rightarrow P^{1} \rightarrow \cdots
$$


such that $\operatorname{Hom}_{S}\left({ }_{S} X, A\right)$ is exact for any flat $S$-module $A$, where all $P^{i}$ are projective $S$-modules. So

$$
{ }_{R} X=0 \rightarrow M^{n} \rightarrow P^{0} \rightarrow P^{1} \rightarrow \cdots
$$

is exact, where all $P^{i}$ are projective $R$-modules.

$$
\begin{aligned}
S \otimes_{R} \operatorname{Hom}_{R}\left({ }_{R} X, Q\right) & \cong \oplus_{i=1}^{n} \operatorname{Hom}_{R}\left({ }_{R} X, Q\right) \\
& \cong \operatorname{Hom}_{R}\left({ }_{R} X, Q^{n}\right) \\
& \cong \operatorname{Hom}_{R}\left({ }_{R} X, S \otimes_{R} Q\right) \\
& \cong \operatorname{Hom}_{R}\left(S \otimes_{S} X, S \otimes_{R} Q\right) \\
& \cong \operatorname{Hom}_{S}\left({ }_{S} X, \operatorname{Hom}_{R}\left(S, S \otimes_{R} Q\right)\right) \\
& \cong \oplus_{i=1}^{n} \operatorname{Hom}_{S}\left({ }_{S} X, S \otimes_{R} Q\right)
\end{aligned}
$$

is exact. Since $S$ is faithfully flat, we get that $\operatorname{Hom}_{R}\left({ }_{R} X, Q\right)$ is exact. By Proposition $2.6(1), M^{n}$ is a $D$-projective $R$-module, and then $M$ is a $D$-projective $R$ module.

Corollary 3.5. Let $S \geq R$ be an excellent extension of $R$. For each $R$-module $M$, we have $\operatorname{Dpd}_{R}(M)=D p d_{S}\left(S \otimes_{R} M\right)$.

Proof. By Corollary 3.2, $D p d_{R}(M) \geq D p d_{S}\left(S \otimes_{R} M\right)$. Now, we assume that $D p d_{S}\left(S \otimes_{R} M\right)=m<\infty$. There is an exact sequence

$$
0 \rightarrow G \rightarrow G_{m-1} \rightarrow \cdots \rightarrow G_{1} \rightarrow G_{0} \rightarrow M \rightarrow 0,
$$

where $G_{i}$ are all $D$-projective $R$-modules. Since $S$ is a free $R$-module, applying $S \otimes_{R}$ - to the above exact sequence, we obtain the exact sequence of $S$-modules:

$$
0 \rightarrow S \otimes_{R} G \rightarrow S \otimes_{R} G_{m-1} \rightarrow \cdots \rightarrow S \otimes_{R} G_{1} \rightarrow S \otimes_{R} G_{0} \rightarrow S \otimes_{R} M \rightarrow 0,
$$

where each $S \otimes_{R} G_{i}$ is a $D$-projective $S$-module by Proposition 3.1. Thus $S \otimes_{R} G$ is also $D$-projective by [13, Theorem 2.4]. From Theorem $3.4, G$ is a $D$-projective $R$-module. So $\operatorname{Dpd}_{R}(M) \leq D p d_{S}\left(S \otimes_{R} M\right)$.

Proposition 3.6. Let $S \geq R$ be an excellent extension of $R$, and let $M$ be an $S$-module. Then $M$ is a $D$-projective $R$-module if and only if $M$ is a D-projective $S$-module.

Proof. $\Rightarrow$ From Proposition 3.1, $S \otimes_{R} M$ is a $D$-projective $S$-module. By Proposition $2.6(1), M$ is a $D$-projective $S$-module, since ${ }_{S} M$ is a direct summand of ${ }_{S}\left(S \otimes_{R} M\right)$ following from Lemma 2.2 .

$\Leftarrow$ Assume that $M$ is a $D$-projective $S$-module, there is an exact sequence of projective $S$-modules:

$$
{ }_{S} Y=\cdots \rightarrow P_{1} \rightarrow P_{0} \rightarrow P^{0} \rightarrow P^{1} \rightarrow \cdots .
$$


Moreover,

$$
{ }_{R} Y=\cdots \rightarrow P_{1} \rightarrow P_{0} \rightarrow P^{0} \rightarrow P^{1} \rightarrow \cdots
$$

is exact with all $P^{i}$ projective $R$-modules, since every $S$-module is an $R$-module and every projective $S$-module is a projective $R$-module. Now we claim that $H m_{R}\left({ }_{R} Y, Q\right)$ is exact for any flat $R$-module $Q$. In fact,

$$
\begin{aligned}
\operatorname{Hom}_{R}\left({ }_{R} Y, Q\right) & \cong \operatorname{Hom}_{R}\left(S \otimes_{S} Y, Q\right) \\
& \cong \operatorname{Hom}_{S}\left(Y, \operatorname{Hom}_{R}(S, Q)\right) \\
& \cong \operatorname{Hom}_{S}\left(Y, S \otimes_{R} Q\right)
\end{aligned}
$$

is exact, since $S \otimes_{R} Q$ is a projective $S$-module. So $M$ is a $D$-projective $R$-module.

Corollary 3.7. Let $S \geq R$ be an excellent extension of $R$. For each $S$-module $M$, we have $\operatorname{Dpd}_{R}(M)=D p d_{S}(M)=D p d_{S}\left(S \otimes_{R} M\right)$.

Proof. We first claim that $D p d_{R}(M) \leq D p d_{S}(M)$. If $D p d_{S}(M)=\infty$, it is trivial. Now we assume that $D p d_{S}(M)=n<\infty$. There is an exact sequence

$$
0 \rightarrow A_{n} \rightarrow \cdots \rightarrow A_{1} \rightarrow A_{0} \rightarrow M \rightarrow 0,
$$

where all $A_{i}$ are $D$-projective $S$-modules. By Proposition 3.6, all $A_{i}$ are $D$ projective $R$-modules. Therefore, $D p d_{R}(M) \leq D p d_{S}(M)$. On the other hand, we have $D p d_{S}(M) \leq D p d_{S}\left(S \otimes_{R} M\right)$ since ${ }_{S} M$ is isomorphic to a direct summand of ${ }_{S}\left(S \otimes_{R} M\right)$. Finally, $D p d_{S}\left(S \otimes_{R} M\right) \leq D p d_{R}(M)$ by Corollary 3.2 .

The left global Ding projective dimension of $R$, denoted by $\operatorname{lgl} D p d(R)$ is defined as the supremum of the $D$-projective dimension of $R$-modules, i.e., $\operatorname{lgl} D p d(R)=$ $\sup \left\{D p d_{R}(M) \mid \forall M \in_{R} \mathcal{M}\right\}$. Now we prove the first main result of this section.

Theorem 3.8. Let $S \geq R$ be an excellent extension of $R$. $\operatorname{lgl} D p d(S)=\operatorname{lglDpd}(R)$.

Proof. By Corollary 3.5, $\operatorname{lgl} D p d(S) \geq \operatorname{lgl} D p d(R)$, and by Corollary 3.7, $\operatorname{lgl} D p d(S) \leq$ $\operatorname{lgl} \operatorname{Dpd}(R)$.

Remark 3.9. We have the similar properties on the D-injective dimensions under change of rings. For instance, let $\theta: R \rightarrow S$ be a ring homomorphism with ${ }_{R} S$ projective. Then $\operatorname{Did}_{S}\left(\operatorname{Hom}_{R}(S, M)\right) \leq \operatorname{Did}_{R}(M)$. Let $S \geq R$ be an excellent extension of $R$ and $\operatorname{lglDid}(R)=\sup \left\{\operatorname{Did}_{R}(M) \mid \forall M \in_{R} \mathcal{M}\right\}$. Then $\operatorname{lglDid}(S)=$ $\operatorname{lgl} \operatorname{Did}(R)$.

Let $\mathcal{F}$ be a class of $R$-modules. $\mathcal{F}^{\perp}$ will denote the right orthogonal class of $\mathcal{F}$, that is, $\mathcal{F}^{\perp}=\left\{M \mid \operatorname{Ext}_{R}^{1}(F, M)=0, \forall F \in \mathcal{F}\right\}$. Analogously, $\perp_{\mathcal{F}}=$ $\left\{M \mid \operatorname{Ext}_{R}^{1}(M, F)=0, \forall F \in \mathcal{F}\right\}$. Following [7], a pair of classes of $R$-modules 
$(\mathcal{A}, \mathcal{B})$ is said to be a cotorsion pair if $\mathcal{A}={ }^{\perp} \mathcal{B}$ and $\mathcal{B}=\mathcal{A}^{\perp}$. A cotorsion pair $(\mathcal{A}, \mathcal{B})$ is said to be hereditary if $\mathcal{A}$ is resolving or, equivalently, $\mathcal{B}$ is coresolving. A cotorsion pair $(\mathcal{A}, \mathcal{B})$ is said to be have enough injective if for any $R$-module $M$ there is an exact sequence $0 \rightarrow M \rightarrow B \rightarrow A \rightarrow 0$ with $B \in \mathcal{B}$ and $A \in \mathcal{A}$. Dually, a cotorsion pair $(\mathcal{A}, \mathcal{B})$ is said to be have enough projective if for any $R$-module $M$ there is an exact sequence $0 \rightarrow B \rightarrow A \rightarrow M \rightarrow 0$ with $B \in \mathcal{B}$ and $A \in \mathcal{A}$. Recall that a ring $R$ is called a Ding-Chen ring if it is left and right coherent, and $F P-i d_{R}\left({ }_{R} R\right)=F P-i d_{R}\left(R_{R}\right)=n$ for some positive integer $n$. The author in $[22$, Theorem 3.6] proved that $\operatorname{Hom}(-,-)$ is right balance by $\mathcal{D P}(R) \times \mathcal{D} \mathcal{I}(R)$ for any Ding-Chen ring $R$. Now we give the new proof for this.

Theorem 3.10. Let $R$ be a Ding-Chen ring. $\operatorname{Hom}(-,-)$ is right balance by $\mathcal{D P}(R) \times \mathcal{D} \mathcal{I}(R)$.

Proof. We first denote the class of all $R$-modules of finite flat dimension by $\mathcal{W}$. By [14, Theorem 3.8] and [10, Corollary 4.6], $(\mathcal{D P}(R), \mathcal{W})$ is a cotorsion pair with enough injective and projective. By [15, Theorem 3.4] and [10, Corollary 4.5], $(\mathcal{W}, \mathcal{D} \mathcal{P}(R))$ is a cotorsion pair with enough injective and projective. By $[22$, Lemma 3.2], $(\mathcal{D P}(R), \mathcal{W})$ and $(\mathcal{W}, \mathcal{D P}(R))$ are hereditary. Then the assertion is true by [9, Theorem 2.2.1].

In the classical homological theory, it is well-known that for any ring $R$, the left projective global dimension is equal to the left injective global dimension, that is, $\lg \operatorname{lpd}(R)=\operatorname{lglid}(R)$. Such result was generalized by Bennis and Mahdou in [3] for Gorenstein homological theory as $\operatorname{lgl} \operatorname{Gpd}(R)=\operatorname{lgl} \operatorname{Gid}(R)$. Similarly, we have

Corollary 3.11. $\operatorname{lgl} \operatorname{Dpd}(R)=\operatorname{lglDid}(R)$ for any Ding-Chen ring.

Proof. It is clear by the above theorem and [9, Corollary 2.2.2].

Now we characterize finite $D$-projective dimension of $R$-modules. For doing this, we first give the following lemma which plays a crucial role.

Lemma 3.12. Let $0 \rightarrow A \rightarrow G_{1} \rightarrow G_{0} \rightarrow M \rightarrow 0$ be an exact sequence with $G_{0}$ and $G_{1} D$-projective. Then there are two exact sequences $0 \rightarrow A \rightarrow P \rightarrow G \rightarrow M \rightarrow 0$ with $P$ projective and $G D$-projective and $0 \rightarrow A \rightarrow H \rightarrow Q \rightarrow M \rightarrow 0$ with $Q$ projective and $H$ D-projective.

Proof. Set $K=\operatorname{Im}\left(G_{1} \rightarrow G_{0}\right)$. Since $G_{1}$ is $D$-projective, there is a short exact sequence $0 \rightarrow G_{1} \rightarrow P \rightarrow G_{1}^{\prime} \rightarrow 0$ with $P$ projective and $G_{1}^{\prime} D$-projective. Consider 
the following pushout diagram:

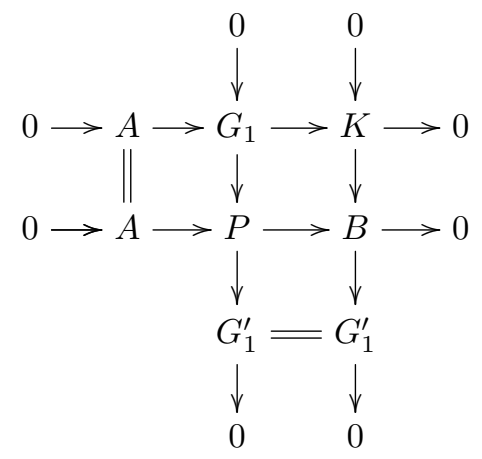

Then consider the following pushout diagram:

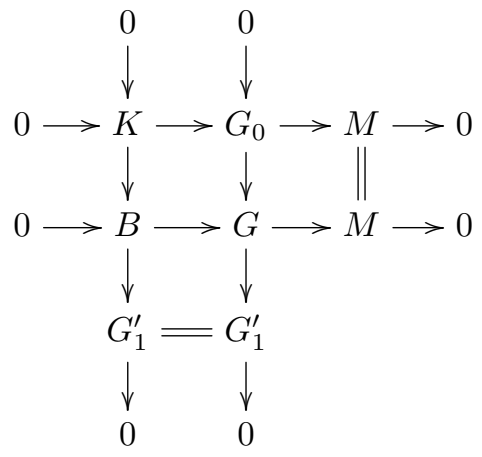

By Proposition 2.6, $G$ is $D$-projective, since $G_{0}$ and $G_{1}^{\prime}$ are $D$-projective. Therefore, we can obtain exact sequence $0 \rightarrow A \rightarrow P \rightarrow G \rightarrow M \rightarrow 0$. Similarly, we use pullbacks and can obtain the other exact sequence.

Theorem 3.13. Let $M$ be an $R$-module and $n$ be a non-negative integer. Then the following are equivalent.

(1) $\operatorname{Dpd}_{R}(M) \leq n$;

(2) For some integer $k$ with $1 \leq k \leq n$, there is an exact sequence $0 \rightarrow P_{n} \rightarrow$ $\cdots \rightarrow P_{1} \rightarrow P_{0} \rightarrow M \rightarrow 0$ such that $P_{i}$ is D-projective if $0 \leq i<k$ and $P_{j}$ is projective if $j \geq k$.

(3) For any integer $k$ with $1 \leq k \leq n$, there is an exact sequence $0 \rightarrow P_{n} \rightarrow$ $\cdots \rightarrow P_{1} \rightarrow P_{0} \rightarrow M \rightarrow 0$ such that $P_{i}$ is D-projective if $0 \leq i<k$ and $P_{j}$ is projective if $j \geq k$.

Proof. $(3) \Rightarrow(2)$ and $(2) \Rightarrow(1)$ are clear.

$(1) \Rightarrow(3)$ Let $0 \rightarrow G_{n} \rightarrow \cdots \rightarrow G_{1} \rightarrow G_{0} \rightarrow M \rightarrow 0$ be an exact sequence with all $G_{i} D$-projective. We prove (3) by induction on $n$. Let $n=1$. Since $G_{1}$ is $D$-projective, there is a short exact sequence $0 \rightarrow G_{1} \rightarrow P_{1} \rightarrow N \rightarrow 0$ with $P_{1}$ 
projective and $N D$-projective. Consider the following pushout diagram:

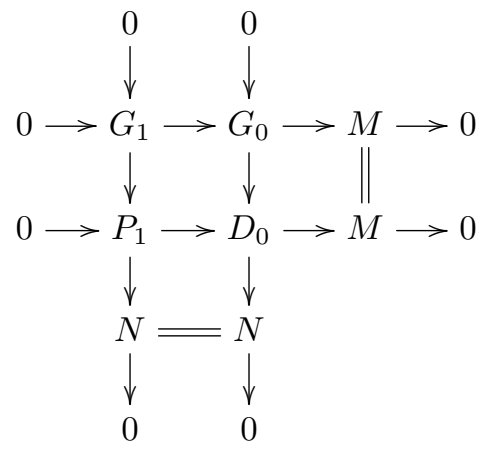

By Proposition 2.6, $D_{0}$ is $D$-projective, since $G_{0}$ and $N$ are $D$-projective. Now assume that $n>1$. Set $A=\operatorname{Ker}\left(G_{0} \rightarrow M\right)$, then $D p d_{R}(A) \leq n-1$. By the induction hypothesis, for any integer $k$ with $2 \leq k \leq n$, there is an exact sequence $0 \rightarrow P_{n} \rightarrow \cdots \rightarrow P_{1} \rightarrow A \rightarrow 0$ such that $P_{i}$ is $D$-projective if $1 \leq i<k$ and $P_{j}$ is projective if $j \geq k$. Therefore, there is an exact sequence $0 \rightarrow P_{n} \rightarrow \cdots \rightarrow P_{1} \rightarrow$ $G_{0} \rightarrow M \rightarrow 0$. Set $B=\operatorname{Ker}\left(P_{1} \rightarrow G_{0}\right)$. For the exact sequence $0 \rightarrow B \rightarrow P_{1} \rightarrow$ $G_{0} \rightarrow M \rightarrow 0$, by Lemma 3.12, there is an exact sequence $0 \rightarrow B \rightarrow P_{1}^{\prime} \rightarrow G_{0}^{\prime} \rightarrow$ $M \rightarrow 0$ with $P_{1}^{\prime}$ projective and $G_{0}^{\prime} D$-projective. Therefore, we get the wanted exact sequence $0 \rightarrow P_{n} \rightarrow \cdots \rightarrow P_{2} \rightarrow P_{1}^{\prime} \rightarrow G_{0}^{\prime} \rightarrow M \rightarrow 0$.

Remark 3.14. (1) In [20, Definition 3.1], the author called a bounded $G_{C}$-projective resolution of $R$-module $M$ a strict $G_{C}$-projective resolution if there is an exact sequence

$$
0 \rightarrow G_{n} \rightarrow G_{n-1} \rightarrow \cdots \rightarrow G_{1} \rightarrow G_{0} \rightarrow M \rightarrow 0
$$

with all $G_{i}$ projective for $i \geq 1$ and $G_{0} G_{C}$-projective. And it is proved that every $R$-module $M$ of finite $G_{C}$-projective dimension always admits a strict $G_{C}$-projective resolution [20, Thereom 3.6]. Using the different method (Theorem 3.20), we can prove that the $R$-module $M$ of finite D-projective dimension has the similar property.

(2) Let $\mathcal{F}$ be a class of $R$-modules. A morphism $\varphi: F \rightarrow M$ of $\mathcal{A}$ is called an $\mathcal{F}$-precover of $M$ if $F \in \mathcal{F}$ and $\operatorname{Hom}\left(F^{\prime}, F\right) \rightarrow \operatorname{Hom}\left(F^{\prime}, M\right) \rightarrow 0$ is exact for all $F^{\prime} \in \mathcal{F} . \varphi$ is called an epic $\mathcal{F}$-precover of $M$ if it is an $\mathcal{F}$-precover and is an epimorphism. If every $R$-module admits an (epic) $\mathcal{F}$-precover, then we say $\mathcal{F}$ is an (epic) precovering class. $M$ is said to have a special $\mathcal{F}$-precover if there is an exact sequence

$$
0 \longrightarrow \mathrm{C} \longrightarrow \mathrm{F} \longrightarrow \mathrm{M} \longrightarrow 0
$$

with $F \in \mathcal{F}$ and $\operatorname{Ext}^{1}(\mathcal{F}, C)=0$. It is clear that $M$ has an epic $\mathcal{F}$-precover if it has a special $\mathcal{F}$-precover. For more details about precovers, readers can refer 
to $[7,9,18]$. The authors in $[13$, Theorem 2.2] proved the following result: If $M$ is an $R$-module with $\operatorname{Dpd}_{R}(M)<\infty$, then $M$ admits a special D-projective precover $\varphi: G \rightarrow M$ where $p d_{R}(\operatorname{Ker} \varphi)=n-1$ if $n>0$ and $\operatorname{Ker} \varphi=0$ if $n=0$. We can use the above theorem to give it a new proof.

Theorem 3.15. Let $M$ be an $R$-module and $n$ be a non-negative integer. Then the following are equivalent.

(1) $\operatorname{Dpd}_{R}(M) \leq n$;

(2) For some integer $k$ with $0 \leq k \leq n$, there is an exact sequence $0 \rightarrow A_{n} \rightarrow$ $\cdots \rightarrow A_{1} \rightarrow A_{0} \rightarrow M \rightarrow 0$ such that $A_{k}$ is D-projective and other $A_{i}$ projective.

(3) For any integer $k$ with $0 \leq k \leq n$, there is an exact sequence $0 \rightarrow A_{n} \rightarrow$ $\cdots \rightarrow A_{1} \rightarrow A_{0} \rightarrow M \rightarrow 0$ such that $A_{k}$ is D-projective and other $A_{i}$ projective.

Proof. $(3) \Rightarrow(2)$ and $(2) \Rightarrow(1)$ are clear.

$(1) \Rightarrow(3)$ Let $0 \rightarrow G_{n} \rightarrow \cdots \rightarrow G_{1} \rightarrow G_{0} \rightarrow M \rightarrow 0$ be an exact sequence with all $G_{i} D$-projective. We prove (3) by induction on $n$. If $n=1$, by Lemma 3.12 , the assertion is true. Now we assume that $n \geq 2$. Set $K=\operatorname{Ker}\left(G_{1} \rightarrow G_{0}\right)$. For the exact sequence $0 \rightarrow K \rightarrow G_{1} \rightarrow G_{0} \rightarrow M \rightarrow 0$, by Lemma 3.12, we get two exact sequences $0 \rightarrow K \rightarrow G_{1}^{\prime} \rightarrow P_{0} \rightarrow M \rightarrow 0$ with $G_{1}^{\prime} D$-projective and $P_{0}$ projective and $0 \rightarrow G_{n} \rightarrow \cdots \rightarrow G_{2} \rightarrow G_{1}^{\prime} \rightarrow P_{0} \rightarrow M \rightarrow 0$. Set $N=\operatorname{Ker}\left(P_{0} \rightarrow M\right)$, then $\operatorname{Dpd}_{R}(N) \leq n-1$. By the induction hypothesis, for any integer $k$ with $1 \leq k \leq n$, there is an exact sequence $0 \rightarrow A_{n} \rightarrow \cdots \rightarrow A_{1} \rightarrow N \rightarrow 0$ such that $A_{k}$ is $D$-projective and other $A_{i}$ projective. Therefore, we get the wanted exact sequence $0 \rightarrow A_{n} \rightarrow \cdots \rightarrow A_{1} \rightarrow P_{0} \rightarrow M \rightarrow 0$. Now we prove the case $k=0$. Set $A=\operatorname{Ker}\left(G_{0} \rightarrow M\right)$, then $\operatorname{Dpd}_{R}(A) \leq n-1$. By the induction hypothesis, there is an exact sequence $0 \rightarrow B_{n} \rightarrow \cdots \rightarrow B_{1} \rightarrow A \rightarrow 0$ such that $B_{1}$ is $D$-projective and other $B_{i}$ projective. So we have an exact sequence $0 \rightarrow B_{n} \rightarrow \cdots \rightarrow B_{1} \rightarrow G_{0} \rightarrow M \rightarrow 0$. Set $B=\operatorname{Ker}\left(B_{1} \rightarrow G_{0}\right)$. For the exact sequence $0 \rightarrow B \rightarrow B_{1} \rightarrow G_{0} \rightarrow M \rightarrow 0$, by Lemma 3.12, we get an exact sequence $0 \rightarrow B \rightarrow P^{\prime \prime} \rightarrow G \rightarrow M \rightarrow 0$ with $G D$-projective and $P^{\prime \prime}$ projective. Hence the exact sequence $0 \rightarrow B_{n} \rightarrow \cdots \rightarrow B_{2} \rightarrow P^{\prime \prime} \rightarrow G \rightarrow M \rightarrow 0$ is as desired.

Remark 3.16. Using the dual arguments, one can obtain the dual versions of Theorem 3.13 and 3.15 on D-injective $R$-modules. 


\section{Strongly $D$-projective modules and strongly $D$-injective modules}

Bennis and Mahdou in [1] introduced three new classes of modules named strongly Gorenstein projective, injective and flat modules which are the special classes of Gorenstein projective, injective and flat modules, respectively. Recall that an $R$ module $M$ is called strongly Gorenstein projective if there exists an exact complex of the form

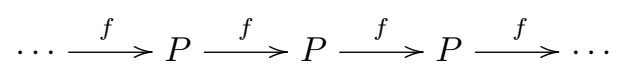

with $P$ projective and such that $M=\operatorname{Ker}(f)$ and the $\operatorname{complex} \operatorname{Hom}_{R}(P, Q)$ is exact for every projective $R$-module $Q$. The strongly Gorenstein injective modules are defined dually. It was proved that a module is Gorenstein projective (respectively, injective) if and only if it is a direct summand of a strongly Gorenstein projective (respectively, injective) module. Recall that the second change of rings theorem [19] as follows: Let $x \in R$ be a central nonzerodivisor in a ring. If $M$ is an $R$-module and $x$ is a nonzerodivisor on $M$, then (1) $p d_{R / x}(M / x M) \leq p d_{R}(M)$; (2) $i d_{R / x}(M / x M) \leq i d_{R}(M)-1$ if $i d_{R}(M) \geq 1$. Bennis and Mahdou in [2] investigate the change of rings theorems for the Gorenstein dimensions. In this section, like the strongly Gorenstein projective and injective modules, we first introduce the strongly Ding projective and injective modules and research their properties. Finally, we consider the second change of rings theorem for Ding projective and injective modules.

Definition 4.1. (1) A left $R$-module $M$ is called strongly $D$-projective if there exists an exact complex of the form

$$
\mathbf{P}=\quad \cdots \stackrel{f}{\longrightarrow} P \stackrel{f}{\longrightarrow} P \stackrel{f}{\longrightarrow} P \stackrel{f}{\longrightarrow} \cdots
$$

with $P$ projective and such that $M=\operatorname{Ker}(f)$ and the complex $\operatorname{Hom}_{R}(\mathbf{P}, Q)$ is exact for every flat $R$-module $Q$.

(2) A left $R$-module $M$ is called strongly $D$-injective if there exists an exact complex of the form

$$
\mathbf{I}=\quad \cdots \stackrel{f}{\longrightarrow} I \stackrel{f}{\longrightarrow} I \stackrel{f}{\longrightarrow} I \stackrel{f}{\longrightarrow} \cdots
$$

with $P$ projective and such that $M=\operatorname{Ker}(f)$ and the complex $\operatorname{Hom}_{R}(E, \mathbf{I})$ is exact for every $F P$-injective $R$-module $E$.

We use $\mathcal{S D P}(R)(\mathcal{S D I}(R)$, respectively) to denote the class of all strongly $D$ projective ( $D$-injective, respectively) $R$-modules. Like the strongly $G$-projective and $G$-injective modules, we have 
Proposition 4.2. (1) $\mathcal{S D} \mathcal{P}(R)$ is closed under direct sums. Every projective module is strongly D-projective module.

(2) $\mathcal{S D I}(R)$ is closed under direct products. Every injective module is strongly D-injective module.

Theorem 4.3. (1) An R-module is D-projective if and only if it is a direct summand of some strongly $D$-projective $R$-module.

(2) An R-module is D-injective if and only if it is a direct summand of some strongly D-injective $R$-module.

Proof. (1) Since strongly $D$-projective $R$-modules are contained by $D$-projective $R$-modules, and the class of $D$-projective $R$-modules is closed under direct summands [24, Corollary 2.7], the assertion can be proved by modifying the proof of [1, Theorem 2.7].

(2) The $D$-injective case is analogous.

Remark 4.4. (1) Since any quasi-frobenius ring is perfect and any $G$-projective $R$ module over a perfect ring is D-projective, by [1, Example 2.5], strongly D-projective modules are not necessarily projective. It is clear that strongly D-projective modules are strongly $G$-projective, and if $R$ is perfect, they coincide with each other. If $G$-projective modules are not necessarily D-projective, by Theorem 4.3, strongly $G$-projective modules are not necessarily strongly D-projective. Therefore,

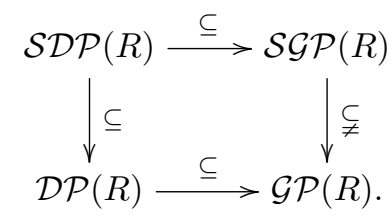

(2) Since any quasi-frobenius ring is noetherian and any G-injective over a noetherian ring is D-injective, by [1, Example 2.5], strongly D-injective modules are not necessarily injective. It is clear that strongly $D$-injective modules are strongly $G$-injective, and if $R$ is noetherian, they coincide with each other. If $G$-injective modules are not necessarily D-injective, by Theorem 4.3, strongly G-injective modules are not necessarily strongly D-injective. Therefore,

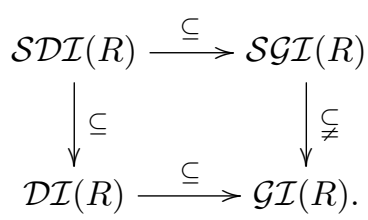

(3) We don't know whether there exists D-projective (D-injective, respectively) module which is not strongly D-projective (D-injective, respectively) module and 
whether there exists $G$-projective (G-injective, respectively) module which is not $D$-projective (D-injective, respectively) module.

Theorem 4.5. For any $R$-module $M$, the following are equivalent.

(1) $M$ is strongly D-projective;

(2) There is a short exact sequence

$$
0 \rightarrow M \rightarrow P \rightarrow M \rightarrow 0
$$

with $P$ projective and $\operatorname{Ext}_{R}^{i>1}(M, Q)=0$ for any flat $R$-module $Q$;

(3) There is a short sequence

$$
0 \rightarrow M \rightarrow P \rightarrow M \rightarrow 0
$$

with $P$ projective and $\operatorname{Ext}_{R}^{i \geq 1}(M, Q)=0$ for any $R$-module $Q$ with finite flat dimension.

Proof. It is easy to prove this from the definition of strongly $G$-projective modules.

We point out that by Theorem 4.5 and [1, Proposition 2.12] the class of finitely generated strongly $D$-projective and the class of finitely generated strongly $G$ projective are the same.

Proposition 4.6. The following are equivalent.

(1) The (strongly) D-projective $R$-module $M$ is flat (projective);

(2) The strongly $G$-projective $R$-module $M$ is flat;

(3) $f d_{R}(M)<\infty$.

Proof. (2) and (3) are equivalent by [1, Corollary 2.11]. Let $M$ be a (strongly) $D$-projective $R$-module. By [5, Lemma 2.4], (3) implies that $M$ is projective.

Proposition 4.7. Let $R$ be a Ding-Chen ring. For any positive integer $n$, the following are equivalent.

(1) $w D(R) \leq n$;

(2) Every strongly $D$-projective $R$-module is flat;

(3) Every strongly D-projective $R$-module is projective.

Proof. By [5, Lemma 2.4], it is clear that (2) and (3) are equivalent. By [5, Proposition 2.14], it just needs to check that every $D$-projective $R$-module is flat when every strongly $D$-projective $R$-module is flat. Since every $D$-projective $R$ module is a direct summand of some strongly $D$-projective $R$-module, it is clear. 
Lemma 4.8. (1) Let

$$
0 \rightarrow N \rightarrow M \rightarrow Q \rightarrow 0
$$

be a short exact sequence with $Q$ projective. $N$ is strongly $D$-projective if and only if $M$ is strongly D-projective.

(2) Let

$$
0 \rightarrow I \rightarrow U \rightarrow V \rightarrow 0
$$

be a short exact sequence with I injective. $U$ is strongly D-injective if and only if $V$ is strongly D-injective.

Proof. (1) First note that since $Q$ is projective, $0 \rightarrow N \rightarrow M \rightarrow Q \rightarrow 0$ is split, hence $M \cong N \oplus Q$. If $N$ is strongly $D$-projective, by Proposition 4.2, $M$ is strongly $D$-projective. Conversely, If $M$ is strongly $D$-projective, there is an exact sequence

$$
0 \rightarrow M \rightarrow P \rightarrow M \rightarrow 0
$$

where $P$ is projective $R$-module and $\operatorname{Ext}_{R}(M, Q)=0$ for any flat $R$-module $Q$. Consider the following pushout diagram:

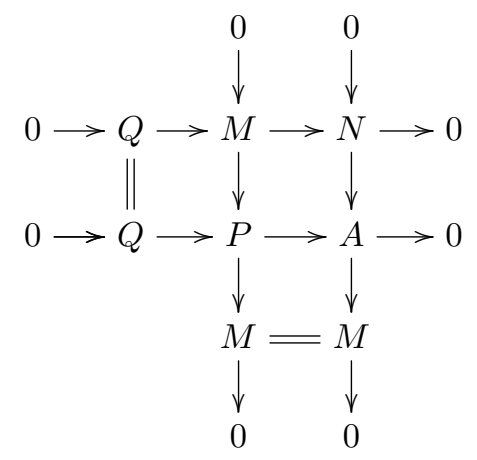

Since $M$ and $N$ are $D$-projective, by Proposition 2.6, $A$ is $D$-projective, and $\operatorname{Ext}_{R}^{1}(A, Q)=0$. So $0 \rightarrow Q \rightarrow P \rightarrow A \rightarrow 0$ is split, therefore, $A$ is projective. Consider the following pullback diagram:

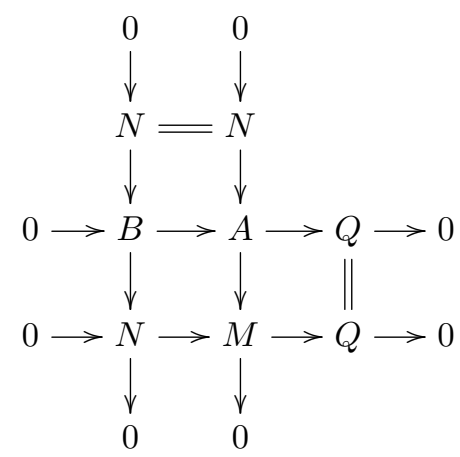


So there is a short exact sequence of the form

$$
0 \rightarrow N \rightarrow B \rightarrow N \rightarrow 0
$$

with $B$ projective. Meanwhile, $\operatorname{Ext}_{R}(N, F)=0$ for any flat $R$-module $F$ since $N$ is $D$-projective. So $N$ is strongly $D$-projective.

Dually, we can prove (2).

It is well-known that the class of all $G$-projective ( $D$-projective, respectively) $R$ modules is projectively resolving [11, Theorem 2.5] ([24, Theorem 2.6], respectively). But for the class of strongly Gorenstein projective $R$-modules it is not true, see [23, P2660]. Like the class of strongly $G$-projective $R$-modules, the class of strongly $D$-projective $R$-modules isn't projectively resolving. For any ring $R$, we have the following result.

Theorem 4.9. The following conditions are equivalent.

(1) The class of the strongly $D$-projective $R$-modules is closed under extensions;

(2) The class of the strongly $D$-projective $R$-modules is projectively resolving;

(3) For any short exact sequence of left $R$-modules $0 \rightarrow G_{1} \rightarrow G_{0} \rightarrow M \rightarrow 0$, where $G_{1}$ and $G_{0}$ are strongly D-projective, if $\operatorname{Ext}_{R}^{1}(M, P)=0$ for any flat $R$-module $P$, then $M$ is strongly $D$-projective;

(4) $\mathcal{D P}(R)=\mathcal{S D P}(R)$.

Proof. (1) $\Rightarrow$ (2) Let $0 \rightarrow A \rightarrow B \rightarrow C \rightarrow 0$ be an exact sequence of left $R$ modules, where $B$ and $C$ are strongly $D$-projective, it is sufficient to prove that $A$ is strongly $D$-projective. Since $C$ is strongly $D$-projective, by Theorem 4.5 there is an exact sequence of left $R$-modules $0 \rightarrow C \rightarrow P \rightarrow C \rightarrow 0$, where $P$ is projective. Consider the following pullback diagram:

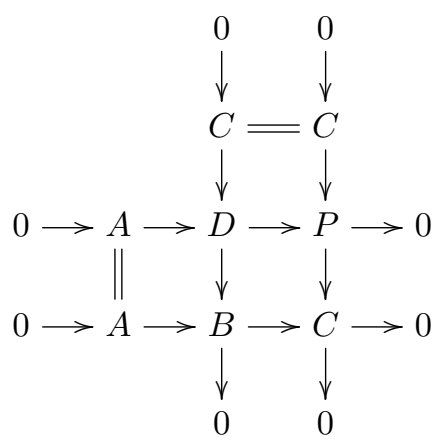

Since $C$ and $B$ are strongly $D$-projective, by (1), $D$ is strongly $D$-projective. By Lemma 4.8, $A$ is strongly $D$-projective, since $P$ is projective.

$(2) \Rightarrow(1)$ Clear. 
(1) $\Rightarrow(3)$ Since $G_{1}$ is strongly $D$-projective, there is an exact sequence of left $R$-modules $0 \rightarrow G_{1} \rightarrow P \rightarrow G_{1} \rightarrow 0$, where $P$ is projective. Consider the following pushout diagram:

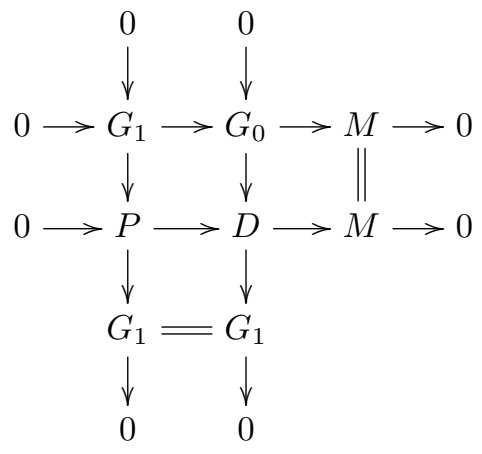

Since $G_{1}$ and $G_{0}$ are strongly Gorenstein projective, so is $D$ by (1). By hypothesis, $\operatorname{Ext}_{R}^{1}(M, P)=0$, thus $0 \rightarrow P \rightarrow D \rightarrow M \rightarrow 0$ is split, i.e., $D \cong P \oplus M$, which is strongly $D$-projective. By Lemma $4.8, M$ is strongly $D$-projective.

(3) $\Rightarrow$ (1) Let $0 \rightarrow A \rightarrow B \rightarrow C \rightarrow 0$ be an exact sequence of left $R$-modules, where $A$ and $C$ are strongly $D$-projective, we prove that $B$ is strongly $D$-projective. Since $C$ is strongly $D$-projective, there is an exact sequence $0 \rightarrow C \rightarrow P \rightarrow C \rightarrow 0$, where $P$ is projective. Consider the following pullback diagram:

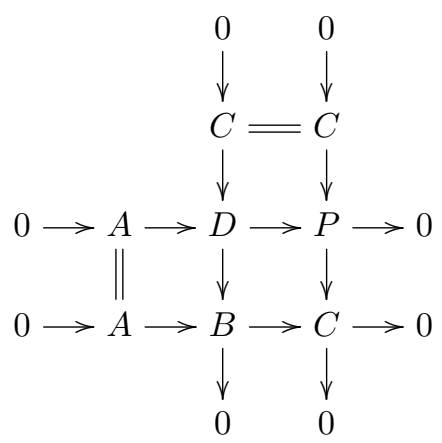

Since the sequence $0 \rightarrow A \rightarrow D \rightarrow P \rightarrow 0$ is exact, where $P$ is projective and $A$ is strongly $D$-projective, by Lemma $4.8, D$ is strongly $D$-projective. On the other hand, for the exact sequence $0 \rightarrow A \rightarrow B \rightarrow C \rightarrow 0$, we have the exact sequence

$$
\operatorname{Ext}_{R}^{i}(C, Q) \rightarrow \operatorname{Ext}_{R}^{i}(B, Q) \rightarrow \operatorname{Ext}_{R}^{i}(A, Q)
$$

for any positive integer $i$ and any flat $R$-module $Q$. Since $A$ and $C$ are strongly $D$-projective, we have that $\operatorname{Ext}_{R}^{i}(C, Q)=\operatorname{Ext}_{R}^{i}(A, Q)=0$ by Theorem 4.5. Thus $\operatorname{Ext}_{R}^{i}(B, Q)=0$. By (3), $B$ is strongly $D$-projective.

$(4) \Rightarrow(2)$ It is trivial. 
$(2) \Rightarrow(4)$ First note that by (2) and [11, proposition 1.4] $\mathcal{S D P}(R)$ is closed under direct summands. Since every $D$-projective $R$-module is a direct summand of some strongly $D$-projective $R$-module, $\mathcal{D P}(R)=\mathcal{S D P}(R)$.

Dually, we have the following.

Theorem 4.10. The following conditions are equivalent.

(1) The class of the strongly D-injective $R$-modules is closed under extensions;

(2) The class of the strongly $D$-injective $R$-modules is injectively resolving;

(3) For any short exact sequence of left $R$-modules $0 \rightarrow M \rightarrow G_{0} \rightarrow G_{1} \rightarrow 0$, where $G_{1}$ and $G_{0}$ are strongly D-injective, if $\operatorname{Ext}_{R}^{1}(E, M)=0$ for any $F P$-injective $R$-module $E$, then $M$ is strongly $D$-injective;

(4) $\mathcal{D} \mathcal{I}(R)=\mathcal{S D I}(R)$.

We are now in a position to consider the second change of rings theorem for $D$-projective and $D$-injective dimensions.

Theorem 4.11. For any $R$-module $M$, let $x \in R$ be a nonzerodivisor on both $M$ and $R$ and be both $R$-regular and $M$-regular. Then

(1) $\operatorname{Dpd}_{R / x}(M / x M) \leq \operatorname{Dpd}_{R}(M)$;

(2) $\operatorname{Did}_{R / x}(M / x M) \leq \operatorname{Did}_{R}(M)-1$ if $\operatorname{Did}_{R}(M) \geq 1$.

Proof. We just prove the first inequality. If $\operatorname{Dpd}_{R}(M)=\infty$, it is trivial. Now we assume $D p d_{R}(M)=n<\infty$ and prove it by induction on $n$. If $D p d_{R}(M)=0$, that is, $M$ is $D$-projective, we need to show $M / x M$ is a $D$-projective $R / x$-module. By Proposition 2.6 and Theorem 4.3, we only assume that $M$ is a strongly $D$-projective $R$-module. By Theorem 4.5, there is a short exact sequence

$$
0 \rightarrow M \rightarrow P \rightarrow M \rightarrow 0
$$

with $P$ projective and $\operatorname{Ext}_{R}^{i \geq 1}(M, Q)=0$ for any flat $R$-module $Q$. Tensoring the above short exact sequence with $R / x$ yields the exact sequence

$$
0 \rightarrow \operatorname{Tor}_{1}^{R}(R / x, M) \rightarrow M / x M \rightarrow P / x P \rightarrow M / x M \rightarrow 0 .
$$

[19, Example 3.1.7], $\operatorname{Tor}_{1}^{R}(R / x, M)=0$. By [17, Theorem 11.65], $\operatorname{Ext}_{R / x}^{i \geq 1}\left(R / x \otimes_{R}\right.$ $M, Q)=\operatorname{Ext}_{R}^{i \geq 1}(M, Q)=0$. Therefore, $M / x M$ is a strongly $D$-projective $R / x$ module from Theorem 4.5. If $\operatorname{Dpd}_{R}(M)=n>0$, there is a short exact sequence

$$
0 \rightarrow K \rightarrow F \rightarrow M \rightarrow 0
$$

with $\operatorname{Dpd}_{R}(K)=n-1$ and $F$ free. By induction, $\operatorname{Dpd}_{R / x}(K / x K) \leq n-1$. Tensoring the short exact sequence $0 \rightarrow K \rightarrow F \rightarrow M \rightarrow 0$ with $R / x$ yields the exact sequence

$$
0 \rightarrow \operatorname{Tor}_{1}^{R}(R / x, M) \rightarrow K / x K \rightarrow F / x F \rightarrow M / x M \rightarrow 0 .
$$


Since $\operatorname{Tor}_{1}^{R}(R / x, M)=0$ and $F / x F$ is a projective $R / x$-module, $D p d_{R / x}(M / x M) \leq$ $1+(n-1)=\operatorname{Dpd}_{R}(M)$.

Corollary 4.12. $D p d_{R[x]}(M[x])=D p d_{R}(M)$.

Proof. It is true by Corollary 3.3 and Theorem 4.11.

\section{References}

[1] D. Bennis and N. Mahdou, Strongly Gorenstein projective, injective, and flat modules, J. Pure Appl. Algebra, 210(2) (2007), 437-445.

[2] D. Bennis and N. Mahdou, First, second and third change of rings theorems for Gorenstein homological dimensions, Comm. Algebra, 38(10) (2010), 38373850 .

[3] D. Bennis and N. Mahdou, Global Gorenstein dimensions, Proc. Amer. Math. Soc., 138(2) (2010), 461-465.

[4] L. W. Christensen, Gorenstein Dimensions, Lecture Notes in Math. Vol. 1747, Springer-Verlag, Berlin, 2000.

[5] N. Ding, Y. Li and L. Mao, Strongly Gorenstein flat modules, J. Aust. Math. Soc., 86(3) (2009), 323-338.

[6] E. E. Enochs and O. M. G. Jenda, Gorenstein injective and projective modules, Math. Z., 220(4) (1995), 611-633.

[7] E. E. Enochs and O. M. G. Jenda, Relative Homological Algebra, de Gruyter Expositions in Mathematics, Vol. 30, Walter de Gruyter, Berlin, 2000.

[8] E. E. Enochs, O. M. G. Jenda, and B. Torrecillas, Gorenstein flat modules, Nanjing Daxue Xuebao Shuxue Bannian Kan, 10(1) (1993), 1-9.

[9] E. E. Enochs and L. Oyonarte, Cover, Envelopes and Cotorsion Theories, Nova Science Publisher, Inc, New York, 2001.

[10] J. Gillespie, Model structures on modules over Ding-Chen rings, Homology, Homotopy Appl., 12(1) (2010), 61-73.

[11] H. Holm, Gorenstein homological dimensions, J. Pure Appl. Algebra, 189 (2004), 167-193.

[12] H. Holm and P. Jørgensen, Semi-dualizing modules and related Gorenstein homological dimensions, J. Pure Appl. Algebra, 205(2) (2006), 423-445.

[13] N. Mahdou and M. Tamekkante, Strongly Gorenstein flat modules and dimensions, Chin. Ann. Math. Ser. B, 32(4) (2011), 533-548.

[14] L. Mao and N. Ding, Relative FP-projective modules, Comm. Algebra, 33(5) (2005), 1587-1602.

[15] L. Mao, and N. Ding, Envelopes and covers by modules of finite FP-injective and flat dimensions, Comm. Algebra, 35(3) (2007), 833-849. 
[16] L. Mao and N. Ding, Gorenstein FP-injective and Gorenstein flat modules, J. Algebra Appl., 7(4) (2008), 491-506.

[17] J. J. Rotman, An Introduction to Homological Algebra, Pure and Applied Mathematics, 85, Academic Press, Inc. New York-London, 1979.

[18] J. Trlifaj, Covers, Envelopes and Cotorsion Theories, Lecture notes for the workshop, Homological methods in module theory, Cortona, September 10$16,2000$.

[19] C. A. Weibel, An Introduction to Homological Algebra, Cambridge Studies in Advanced Mathematics, 38, Cambridge University Press, Cambridge, 1994.

[20] D. White, Gorenstein projective dimension with respect to a semidualizing module, J. Commut. Algebra, 2(1) (2010), 111-137.

[21] W. Xue, On almost excellent extensions, Algebra Colloq., 3(2) (1996), 125-134.

[22] G. Yang, Homological properties of modules over Ding-Chen rings, J. Korean Math. Soc., 49(1) (2012), 31-47.

[23] X. Yang and Z. Liu, Strongly Gorenstein projective, injective and flat modules, J. Algebra, 320(7) (2008), 2659-2674.

[24] G. Yang, Z. Liu and L. Liang, Ding projective and Ding injective modules, Algebra Colloq., 20(4) (2013), 601-612.

\section{Chaoling Huang}

College of Mathematics and Statistics

Hubei Normal University

Huangshi 435002, China

e-mail: clhuang1978@live.cn

\section{Tongsuo Wu}

Department of Mathematics

Shanghai Jiaotong University

Shanghai, 200240, China

e-mail: tswu@sjtu.edu.cn 